\title{
Energy Conservation in Reliable Wireless Sensor Networks
}

\author{
Fatma Othman ${ }^{\ddagger}$, Nizar Bouabdallah ${ }^{\ddagger}$ and Raouf Boutaba \\ $\left.{ }^{\ddagger}\right)$ INRIA-IRISA, Campus de Beaulieu 35042, Rennes, France. \\ (đ) School of Computer Science, University of Waterloo ; 200 University Ave. W., Waterloo, ON, Canada. \\ E-Mail: \{fatma.othman,nizar.bouabdallah\}@inria.fr ; rboutaba@uwaterloo.ca
}

\begin{abstract}
Energy-efficiency is one of the major concerns in wireless sensor networks since it impacts the network lifetime. In this paper, we investigate the relationship between sensor network performance, particularly its lifetime, and the number of active reporting nodes $N$ by using both analytical and simulation approaches. We first demonstrate that decreasing the number of reporting nodes increases the number of reports that need to be sent to the sink, in order to achieve the desired information reliability regarding the detected event. On the other hand, we show that reducing the number of reporting nodes alleviates the energy wastage due to collisions. Based on this tradeoff, and as a main contribution, we derive the optimal number of reporting nodes $N_{\text {opt_energy }}$ that minimizes the energy consumed to report reliably the occurrence of an event. In other words, we prove that limiting the reporting tasks of a detected event to a small subset of sensor nodes (i.e., $N_{\text {opt energy }}$ ), instead of using all the sensor nodes in the event area, enables significant energy conservation.
\end{abstract}

\section{INTRODUCTION}

Energy-efficiency is a critical issue in wireless sensor networks (WSNs) due to the limited capacity of the sensor nodes' batteries [1]. Once a WSN is in place, its lifetime must last as long as possible based on the initially provided amount of energy. Consequently, techniques minimizing energy-consumption are required to improve the network lifetime. A widely employed mechanism is to schedule sensor nodes activity so that redundant nodes enter the sleep mode as often as possible [2]. Based on this concept, several energyefficient MAC protocols [3] [4] and energy-efficient routing protocols [5] have been proposed in the literature.

So far, various studies addressed the energy optimization issue without considering the impact of the number of reporting nodes on the WSN performance. In other words, how the network lifetime evolves with respect to the number of active reporting nodes. Our work is motivated by the results in [6], which highlight the significant energy conservation that could be achieved when spatial correlation is exploited to reduce the number of redundant transmitted packets in the network. Specifically, [6] proposed a MAC protocol that reduces the number of transmitted packets regarding an observed event, by limiting the reporting tasks to a small number of sensor nodes, hence benefiting from the spatial correlation among the densely deployed sensor nodes within the event area.

In other words, [6] shows that using a small subset of the nodes (called representative nodes) rather than all the sensor nodes in the event area, to report the detected event reduces considerably the energy consumption. Indeed, limiting the number of reporting nodes alleviates the energy wastage caused by collisions, idle listening and redundant packet transmission. In the optimal case, only one node will be allowed to report a detected event. In such case, collisions, idle listening and redundant packet transmission are totally eliminated. But, such choice may not guarantee the required reliability since only one report is received by the sink regarding the observed event. In view of this, authors in [6] determine, using the spatial correlation among sensor nodes, the minimum number of representative nodes $N_{\text {min }}$ that need at least to be activated in order to comply with the required data reliability at the sink. Accordingly, each node, among the $N_{\min }$ representative ones, needs to transmit one report only to fulfill the reliability requirement. In this case, the optimal energy consumption in the network is achieved when only $N_{\min }$ reporting nodes are activated while the remaining nodes undergo the sleep mode.

As a key distinguishing feature from [6], in our study, each representative node will be allowed to transmit as much packets as needed to attain the desired reliability. Our aim is to introduce additional flexibility to select among the reporting nodes, which may lead to supplementary energy conservation. Indeed, as a first main contribution of our paper, we demonstrate that, according to our method, the required reliability could be maintained even if the number of active reporting nodes $N$ is less than minimum boundary $N_{\text {min }}$ obtained in [6]. However, this requires more than $N_{\text {min }}$ reports to be transmitted to the sink by the $N<N_{\text {min }}$ active reporting nodes, since the correlation among the transmitted data increases when the number of reporting nodes decreases. In this regard, additional energy may be required to report reliably the detected event.

On the other hand, reducing the number of reporting nodes beyond $N_{\text {min }}$ decreases the energy wastage due to collisions and idle listening. Clearly, a tradeoff exists between these opposite requirements to minimize energy consumption (i.e., the reduction of the energy wastage caused by collisions and idle listening when reducing $N$ beyond $N_{\min }$, at the expense of the increase of the number of reports that need to be sent to the sink to attain the desired reliability). In this paper, we analyze this tradeoff. Specifically, we determine the optimal number of reporting nodes $N_{\text {optenergy }}$ that minimizes the energy consumption in reliable WSNs. As a second main contribution of our paper, we show that $N_{\text {opt_energy }}<N_{\min }$, 


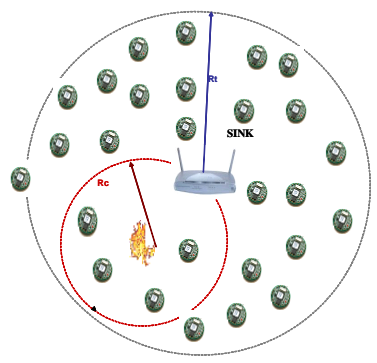

Fig. 1. Example of a sensor network.

which proves that our method does not only introduce more flexibility to attain the desired reliability but it also enables further energy conservation.

The remaining of this paper is structured as follows. Section II presents the general problem statement. In section III we derive the average number of reports $R(N)$ required to report reliably an event as a function of the number of reporting nodes $N$. In section IV, we derive the optimal number of reporting nodes $N_{\text {opt energy }}$ that minimizes the energy consumption in the network. Finally, conclusions are drawn in section $\mathrm{V}$

\section{PRoblem Statement}

Let us consider a WSN as depicted in Fig. 1. In essence, a WSN ensures the supervision of a given area by the use of a sink node, which collects reports from the network. In this analysis we consider event detection driven wireless sensor applications. In other words, communications are triggered by the occurrence of a pre-specified type of events. Once an event occurs, it has to be reported to the sink by the sensor nodes. In such network, sensor nodes, within an event radius $R_{c}$, are the sources (i.e., reporting nodes) for the detected event. Recall that sensor nodes are characterized by their coverage range $R_{c}$ (i.e., sensing range) and transmission range $R_{t}$ as shown in Fig. 1.

We denote by $N_{t o t}$ the total number of sensor nodes within the event area. Then, $N\left(N=1, \ldots, N_{t o t}\right)$ represents the number of active nodes allowed to report that event. The $N$ reporting nodes keep generating reports until the required event detection reliability $R(N)$ is achieved. The desired event reliability, $R(N)$, is the number of data packets required by the sink to consider the event as reliable [7]. Once the sink node receives $R(N)$ reports, it asks the sensor nodes to stop the event reporting.

In this study, we aim at analyzing the impact of the number of active reporting nodes $N$ on the WSN energy consumption. The basic idea behind our proposal is to let some potential reporting nodes enter a sleep mode. In the extreme case, we only let one sensor node $(N=1)$ report a detected event. Furthermore, we evaluate the average number of reports $R(N)$ and the associated energy required to report reliably that event.
III. RELATIONSHIP BETWEEN INFORMATION RELIABILITY AND THE NUMBER OF REPORTING NODES

In this section, we extend the work in [6] to derive the number of reports $R(N)$ required to report reliably an event given that the number of active reporting nodes is $N$. This consists in calculating the minimal number of reports $R(N)$ that need to be sent to the sink by the $N$ active reporting nodes in order to not exceed a predefined tolerable information distortion $D_{\max }$. The event reporting operation is considered reliable only when the distortion between the event source $S$ and its estimation at the sink becomes less than $D_{\max }$.

In [6], the authors provide an expression of the observed information distortion at the sink $D$ when each node among the $N$ representative ones (out of the $N_{t o t}$ sensor nodes in the event area) transmits only one report to the sink. Accordingly, the distortion can be written as follows:

$$
\begin{aligned}
D(N)=\sigma_{S}^{2}- & \frac{\sigma_{S}^{4}}{N\left(\sigma_{S}^{2}+\sigma_{N}^{2}\right)}\left(2 \sum_{i=1}^{N} \rho_{(s, i)}-1\right) \\
& +\frac{\sigma_{S}^{6}}{N^{2}\left(\sigma_{S}^{2}+\sigma_{N}^{2}\right)^{2}} \sum_{i=1}^{N} \sum_{j \neq i}^{N} \rho_{(i, j)}
\end{aligned}
$$

where

- $\sigma_{S}^{2}$ and $\sigma_{N}^{2}$ are the variance of the event information $S_{i}$ and the observation noise $N_{i}$ of each sensor node $n_{i}$ $(i=1, . ., N)$, respectively.

- $\rho_{(s, i)}$ denotes the correlation coefficient between the event source located at coordinate $s$ and the sensor node $n_{i}$ $(i=1, \ldots, N)$.

- $\rho_{(i, j)}$ denotes the correlation coefficient between nodes $n_{i}$ and $n_{j}(i, j=1, \ldots, N)$.

To derive (1), the observation noise $N_{i}$ of each sensor node $n_{i}$ is modeled as i.i.d Gaussian random variable of zero mean and variance $\sigma_{N}^{2}$. Moreover, the event information $S_{i}$ sensed by the node $n_{i}$, which is an observation of the original event source $S$, is modeled as a joint Gaussian random variable (JGRV) as follows:

$$
\begin{aligned}
& E\left\{S_{i}\right\}=0 \quad \operatorname{var}\left\{S_{i}\right\}=\sigma_{S}^{2}, i=1, \ldots, N \\
& \rho_{(i, j)}=\frac{E\left[S_{i}, S_{j}\right]}{\sigma_{S}^{2}}=e^{-\left(d_{(i, j)} / \theta_{1}\right)}, \text { for } \theta_{1}>0
\end{aligned}
$$

where $d_{(i, j)}$ denotes the distance between nodes $n_{i}$ and $n_{j}$. We note that, in this case, the correlation coefficient $\rho_{(i, j)}$ between the sensor observations is estimated using the Power Experimental model [8].

Hence, $D$ simply measures the distortion between the original event $S$ and its estimation at the sink obtained through the $N$ observations $X_{i}=S_{i}+N_{i}$. Based on the expression of $D$, the authors in [6] derive the minimum number $N_{\min }$ of reporting nodes that need to be activated among the $N_{t o t}$ potential ones in order to not exceed the tolerable information distortion $D_{\max }$. In this case, receiving $N_{\min }$ reports at the sink, i.e., one report from each sensor node, is sufficient to attain the desired reliability. 
As mentioned before, unlike the work in [6], in our study, we allow each reporting node to transmit as much packets as needed to attain the desired reliability. Doing so, the required reliability could be achieved even if the number of active reporting nodes $N<N_{\min }$. However this would imply probably more than $N_{\min }$ reports to be received at the sink level in order to fulfill the reliability requirements. Indeed, the correlation among the transmitted data by the WSN increases when the number of reporting nodes decreases. From this perspective, additional energy could be required to report reliably an event since more reports need to be sent.

However, activating only $N<N_{\min }$ sensor nodes reduces the energy wastage due to collisions and idle listening. There must be an optimal value of $N=N_{\text {opt_energy }} \in\left[1, \ldots, N_{\min }\right]$ that achieves the above-mentioned tradeoff, i.e., that minimizes the energy required to report reliably an event. Henceforth, our aim is to demonstrate that $N_{\text {opt_energy }}<N_{\min }$. In doing so, we prove, as it will be shown in section IV, that our proposal enables further energy conservation when compared to [6], as well as additional flexibility to attain the desired reliability.

In what follows, we derive a generalized expression of the distortion $D(N, r)$ associated with our proposal. In this case, $D(N, r)$ depends on both the number of active reporting nodes $N$ and the total number of reports $r$ that they transmit to the sink. Recall that in [6], the expression of the distortion (see (1)) is derived for the particular case $r=N$. Using the same model for the information collection and the same assumptions as in [6], $D(N, r)$ can be expressed as follows:

$$
\begin{aligned}
D(N, r)=\sigma_{S}^{2} & -\frac{\sigma_{S}^{4}}{r\left(\sigma_{S}^{2}+\sigma_{N}^{2}\right)}\left(2 \sum_{k=1}^{r} \rho_{(s, n(k))}-1\right) \\
& +\frac{\sigma_{S}^{6}}{r^{2}\left(\sigma_{S}^{2}+\sigma_{N}^{2}\right)^{2}} \sum_{k=1}^{r} \sum_{m \neq k}^{r} \rho_{(n(k), n(m))}
\end{aligned}
$$

where $n(k)$ denotes the coordinate of the sensor node that transmits the $k^{\text {th }}$ report. We note that in our study we use the same CSMA/CA-based DCF MAC protocol proposed in [6]. According to the CSMA/CA mechanism, all the $N$ competing reporting nodes have equal probability to access the medium. In this regard, the node that transmits the $k^{t h}$ report (i.e., $n(k)$ ) can be with equal probability one of the $N$ reporting nodes. In other words, $\operatorname{Pr}\left\{n(k)=n_{i}\right\}=\frac{1}{N}, \forall i=1, \ldots, N$. Hence, we get

$$
\begin{aligned}
& \rho_{(s, n(k))}=\frac{1}{N} \sum_{i=1}^{N} \rho_{(s, i)}, \quad \forall k=1, \ldots, r . \\
& \rho_{(n(k), n(m))}=\frac{1}{N^{2}} \sum_{i=1}^{N} \sum_{j=1}^{N} \rho_{(i, j)}, \quad \forall k, m=1, \ldots, r .
\end{aligned}
$$

where $i$ and $j(i, j=1, \ldots N)$ are respectively the coordinates of the sensor nodes $n_{i}$ and $n_{j}$. Substituting (4) in (3), the distortion $D(N, r)$ can be therefore written as follows:

$$
\begin{aligned}
D(N, r)=\sigma_{S}^{2} & -\frac{\sigma_{S}^{4}}{r\left(\sigma_{S}^{2}+\sigma_{N}^{2}\right)}\left(2 \frac{r}{N} \sum_{i=1}^{N} \rho_{(s, i)}-1\right) \\
& +\frac{\sigma_{S}^{6}}{r\left(\sigma_{S}^{2}+\sigma_{N}^{2}\right)^{2}} \frac{r-1}{N^{2}} \sum_{i=1}^{N} \sum_{j=1}^{N} \rho_{(i, j)}
\end{aligned}
$$

Figure 2 plots the distortion evolution according to both our method (i.e., using (5)) and work in [6] (i.e., using (1)). The distortion is plotted as a function of the number of transmitted reports $r$ by the $N$ active reporting nodes.

Let us first focus on the results generated using our method. In this case, the distortion is presented for two values of $N$ (i.e., $N=10$ and $N=40$ ). Based on the obtained curves two main observations can be made:

- First, the figure shows that for a given $N$, the observed distortion at the sink decreases logically with the increase of $r$ since the sink receives more information from the event area. This distortion becomes relatively constant when the number of transmitted reports is large. Indeed, in this case, the transmitted data to the sink is highly redundant.

- Second, the same distortion level can be achieved by different values of $N$. But, in this case, the lower is the value of $N$, the greater is the number of reports $R(N)$ required to attain the same reliability. Indeed, the correlation among the transmitted information increases when $N$ decreases. To illustrate this, assume the maximal tolerable distortion $D_{\max }$ is equal to 8 . To achieve this, at least $r=16$ reports need to be transmitted when we activate only $N=10$ reporting nodes among the $N_{\text {tot }}=50$ existing sensor nodes in the event area, whereas $r=6$ reports are sufficient when $N$ is set equal to 40 . To gain insight regarding this finding, Fig.3 represents the minimum number of reports $R(N)$ that need to be sent to the sink to achieve a certain distortion $D_{\max }$, as a function of the number of reporting nodes. As explained previously, we can see that $R(N)$ decreases with $N$.

Let us now consider the results regarding the method introduced in [6], which can be seen as a particular case where $r=N$. As expected, we can observe in Fig. 2 that the obtained curve intersects those with $N=40$ and $N=10$ when $r=40$ and $r=10$, respectively. Moreover, we can see that the distortion decreases logically with the number of transmitted reports (i.e., the number of reporting nodes). As stated before, this method entails a lower bound on the number of reporting nodes that need at least to be activated in order to respect the tolerable distortion. For instance, considering again $D_{\max }=8$, implies activating at least $N_{\min }=12$ sensor nodes to fulfill the distortion requirement. According to this method, activating only $N_{\min }$ reporting nodes allows the optimal energy consumption.

Note that this same level of reliability can be achieved for $N<N_{\min }$ when considering our method (see Fig. 3). In this regard, our scheme introduces further flexibility to achieve the 


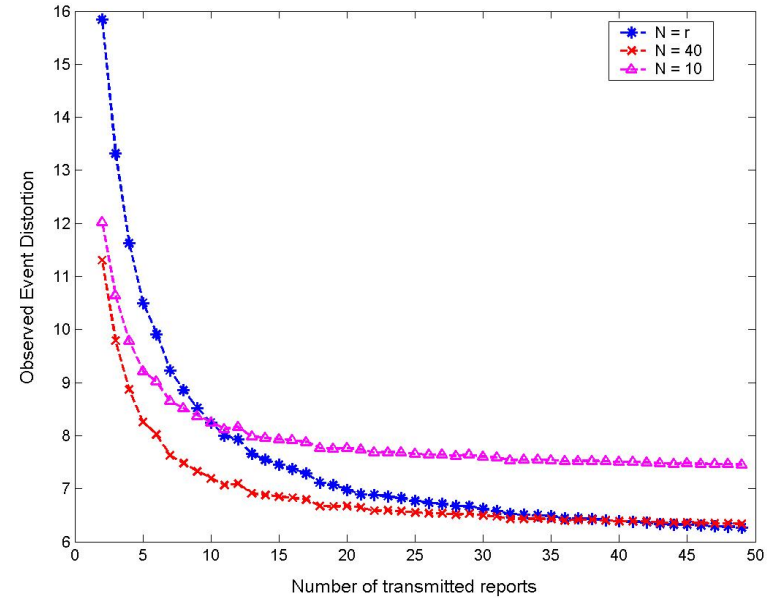

Fig. 2. Average distortion as a function of the number of transmitted reports considering different values of active reporting nodes.

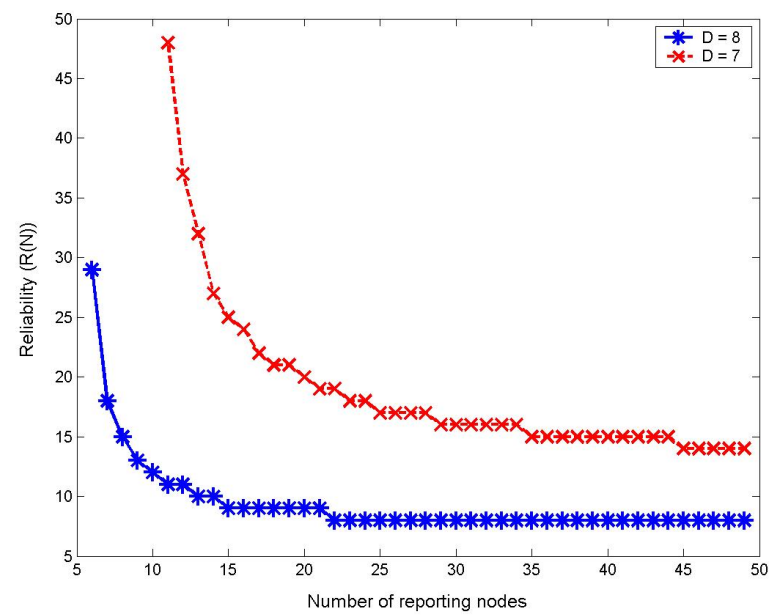

Fig. 3. Average reliability as a function of the number of reporting nodes for different distortion values.

desired distortion at the sink. In the remainder of this paper, we will demonstrate the interest of such flexibility. We will prove that the minimum energy consumption in reliable WSNs can be achieved for $N_{\text {opt_energy }}<N_{\min }$.

\section{Performance Evaluation}

In this section, we analyze the impact of the reporting nodes on the WSN performance by conducting simulations on NS-2.

In our simulations, the sensor nodes are randomly deployed in the sensor field. The average number of nodes that senses an occurring event is $N_{t o t}$, where $N_{t o t}$ is set equal to 50 . Hence, the number of active reporting nodes $N$ varies between 1 and $N_{t o t}=50$. We use the same IEEE 802.11 DCF-based MAC protocol proposed in [6] to arbitrate the access among reporting nodes. The parameters setting in our experiments are listed in table I.

Let us first focus on the impact of $N$ on the average amount of energy consumed by the network to report an event (i.e., to

\begin{tabular}{|l|l|}
\hline Communication range & $40 \mathrm{~m}$ \\
\hline Sensing range & $30 \mathrm{~m}$ \\
\hline Packet length & 30 bytes \\
\hline IFQ length & 65 packets \\
\hline Transmit power & $0.660 \mathrm{~W}$ \\
\hline Receive power & $0.395 \mathrm{~W}$ \\
\hline Idle power & $0.035 \mathrm{~W}$ \\
\hline Sleep power & $0.035 \mathrm{~W}$ \\
\hline Initial network energy & $100 \mathrm{~J}$ \\
\hline
\end{tabular}

TABLE I

SimUlation PARAMETERS

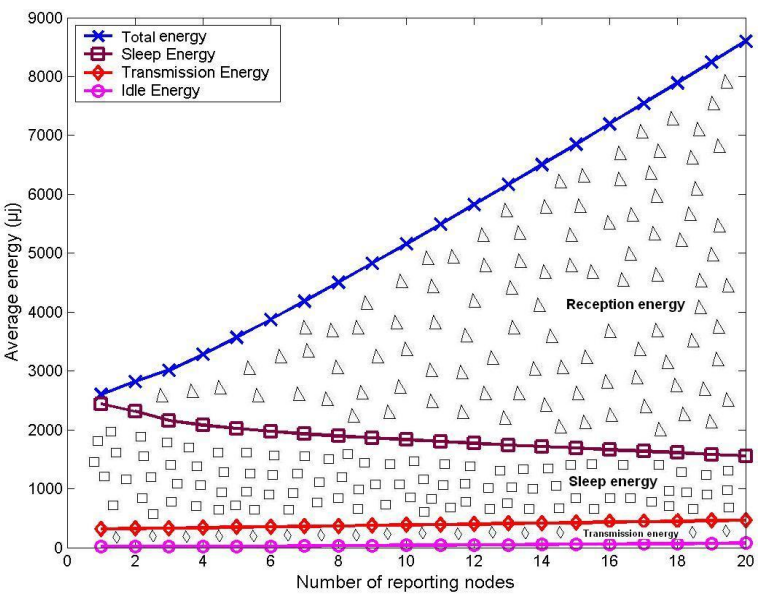

Fig. 4. The average amount of energy consumed by the network to transmit a report.

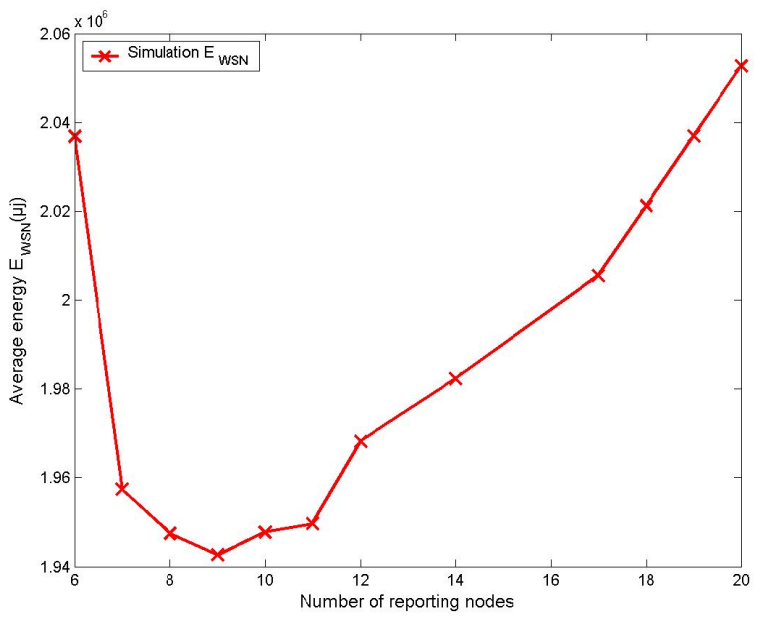

Fig. 5. The average amount of energy consumed by the WSN per unit of time. 
send one report). Figure 4 shows that this amount of energy is monotonically rising with $N$. This monotonous increase is mainly due to two factors. First, increasing $N$ amplifies the wasted energy due to collisions. Moreover, increasing $N$ means waking up more sensor nodes within the event radius $R_{c}$. Doing so, the total amount of energy consumed by the network in the reception of the signaling messages (RTS, CTS, ACK) increases considerably.

According to these results, we can see that the optimal number of active reporting nodes that enables the minimal energy consumption when sending a report is $N=1$. In this case, the wasted energy due to both collisions and idle listening is avoided.

However, a smaller energy to send a report does not mean necessarily that the energy required to report reliably an event is reduced. Indeed, reducing the number of reporting nodes $N$ increases the number of reports $R(N)$ that need to be transmitted to the sink in order to achieve the desired reliability (see Fig. 3). Hence, the optimal energy consumption in such reliable WSNs is a tradeoff between the above-mentioned requirements as shown in Fig. 5.

Figure 5 represents the average amount of energy consumed per unit of time by the WSN, denoted henceforth by $E_{W S N}(N)$, for varying number of reporting nodes $N$. In our simulations, we assume that the rate of event occurrence is $M=5$ (i.e., it occurs 5 events per unit of time). We assume that the maximal tolerable distortion at the sink is $D_{\max }=$ 8. Figure 5 shows that the minimal energy consumption is obtained when only $N_{\text {opt_energy }}=9$ reporting nodes are activated, whereas the remaining ones undergo the sleep mode. Based on this result, we can conclude the following:

- Using a small subset of the nodes rather than all the sensor nodes in the event area to report reliably an event reduces considerably the energy consumption. In our case, the optimal energy consumption is achieved when only $N=9$ out of the $N_{t o t}=50$ sensor nodes are activated.

- A second interesting finding is that $N_{\text {opt_energy }}=9<$ $N_{\min }=12$. Recall that [6] stipulates that at least $N_{\min }=$ 12 sensor nodes should be activated in order to comply with the reliability requirement. Our scheme relaxes this constraint but at the expense of increasing the number of reports' transmission. Yet our scheme reduces the energy consumption as $E_{W S N}(N=9)<E_{W S N}(N=12)$. Consequently, we can state that our scheme does not only introduce more flexibility to attain the desired reliability, but it also enables further energy conservation.

Finally, Fig. 6 plots the network lifetime evolution as a function $N$. Similar to $E_{W S N}$, the maximum network lifetime is obtained when only $N_{\text {opt energy }}=9$ reporting nodes are activated. This result highlights again that limiting the reporting tasks to a small subset of sensor nodes instead of using all the potential ones in the event area enables great energy saving. The maximal gain is obtained for $N_{\text {opt_energy }}=9$. In this regard, our proposal improves the network lifetime when compared to [6], where the maximal network lifetime was

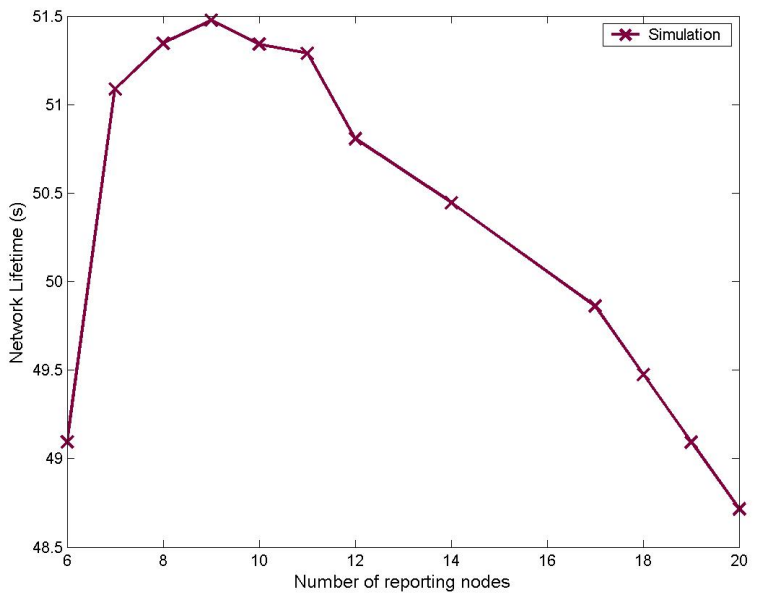

Fig. 6. Sensor network lifetime.

obtained for $N_{\min }=12$. Note that, for the sake of simplicity, we presented the results for the case $D_{\max }=8$ only. Similar results are however obtained for other values of $D_{\max }$. For instance, when $D_{\max }=7$, we have again $N_{\text {opt_energy }}<$ $N_{\text {min }}$, where $N_{\text {opt_energy }}=15$ and $N_{\min }=20$.

\section{CONClusion}

In this paper, we have studied the relationship between the energy consumption and the number of reporting nodes in reliable wireless sensor networks. We first analyzed the impact of the number of reporting nodes on the number of required reports to comply with the desired reliability by extending work in [6]. Based on this analysis, we derived the average energy needed to report reliably an event. We have demonstrated that by limiting the reporting tasks to a small subset of sensor nodes $N_{\text {opt_energy }}$, while the remaining potential ones undergo the sleep mode, we can achieve great energy savings.

\section{REFERENCES}

[1] I. Akiyldiz, W. Su, Y. Sankarasubramaniam and E. Cayirci, A survey on sensor networks, IEEE Communication Magazine, Vol. 40, Issue 8, pp 102 - 114, August 2002.

[2] S. Singh and C.S. Raghavendra, PAMAS: Power Aware Multi-Access Protocol with Signaling for Ad Hoc Networks, ACM Computer Communication. Review., pp. 5-26, July 1998.

[3] M. Miller and N. Vaidya, A mac protocol to reduce sensor network energy consumption using a wake-up radio, IEEE Transactions on Mobile Computing, Vol. 4, Issue 3, pp 228 - 242, May-June 2005.

[4] W. Ye, J. Heidemann, and D. Estrin, Medium access control with coordinated adaptive sleeping for wireless sensor networks, IEEE/ACM Transactions on Networking, vol. 12, no. 3, pp. 493-506, Jun. 2004

[5] J. Chang and L. Tassiulas, Maximum lifetime routing in wireless sensor networks, IEEE/ACM Transactions On Networking, vol. 12, Issue 4, pp 609- 619, August 2004.

[6] M. C. Vuran, and I. F. Akyildiz, Spatial correlation-based collaborative medium access control in wireless sensor networks, IEEE/ACM Transactions On Networking, vol 14, Issue 2, pp 316 - 329, April 2006.

[7] O. B. Akan end I. F. Akyildiz, Event-to-Sink Reliable Transport for Wireless Sensor Networs, IEEE/ACM Transactions on Networking, vol. 13, no. 5, pp. 1003-1016, October 2005.

[8] G. L. Suber, Principles of Mobile Communication, Norwell, MA: Kluwer, 2001. 